\section{AIDS tem cor ou raça? Interpretação de dados e formulação de políticas de saúde no Brasil}

\author{
Does AIDS have a race or color? Data interpretation \\ and health policymaking in Brazil
}

\author{
${ }^{1}$ Instituto de Filosofia \\ e Ciências Sociais, \\ Universidade Federal do \\ Rio de Janeiro, \\ Rio de Janeiro, Brasil. \\ 2 Instituto Oswaldo Cruz, \\ Fundação Oswaldo Cruz, \\ Rio de Janeiro, Brasil. \\ 3 Casa de Oswaldo Cruz, \\ Fundação Oswaldo Cruz, \\ Rio de Janeiro, Brasil. \\ ${ }^{4}$ Centro de Informação \\ Científica e Tecnológica, \\ Fundação Oswaldo Cruz, \\ Rio de Janeiro, Brasil. \\ 5 Escola Nacional de \\ Saúde Pública Sergio Arouca, \\ Fundação Oswaldo Cruz, \\ Rio de Janeiro, Brasil. \\ 6 Departamento de \\ Antropologia, \\ Museu Nacional, \\ Rio de Janeiro, Brasil. \\ Correspondência \\ S. Monteiro \\ Laboratório de Educação \\ em Ambiente e Saúde. \\ Departamento de Biologia, \\ Instituto Oswaldo Cruz, \\ Fundação Oswaldo Cruz. \\ Av. Brasil 4365 \\ Rio de Janeiro, RJ \\ 21045-900, Brasil. \\ msimone@ioc.fiocruz.br
}

\begin{abstract}
Over the last few years we have observed a growing emphasis on a supposed relationship between the AIDS epidemic and the "black population" in Brazil. After undertaking an analysis of the national data base of HIVIAIDS in Brazil, this study examines the sociopolitical context in which public policy with a focus on "race" has been defined. We argue that questions related to the quality of the data, the structuring of the information system itself and the use and interpretation of this information are all essential elements for understanding the process underway. Specifically we aim to show that the available epidemiological data are not sufficient to warrant the interpretation that there is in fact a relationship between the "black population" and AIDS in the country. We stress that the emphasis on this supposed association is part of a more general process of construction of the field of the "health of the black population" in recent years and that this is related to interrelationships between political activism and the State which go far beyond the field of health.
\end{abstract}

Acquired Immunodeficiency Syndrome; Public Policies; Racism
Peter H. Fry ${ }^{1}$

Simone Monteiro 2

Marcos Chor Maio 3

Francisco I. Bastos 4

Ricardo Ventura Santos 5,6

\section{Introdução}

No dia 1o de dezembro de 2004, Dia Mundial de Luta Contra a AIDS, O Globo e outros jornais noticiaram o lançamento do Boletim Epidemiológico - AIDS de 2004, divulgado pelo Ministério da Saúde. Apesar de quase todas as manchetes terem enfatizado o crescimento da epidemia entre mulheres e "negros", o então Diretor do Programa Nacional de DST/AIDS (PN-DST/AIDS), Pedro Chequer, afirmou: "a AIDS não é uma doença [sic] associada à raça negra, tanto que a maioria dos casos registrados é de gente branca, [...] a população negra de mais baixa escolaridade é mais desinformada e, portanto, mais exposta à doença [sic]” 1 (p. 14). A então presidente da organização não-governamental (ONG) Arco-Íris, Ana Paula Prado, ofereceu interpretação similar: "a doença [sic] começou a ter a cara do Brasil. Ataca pessoas pobres, de baixa instrução e nos lugares mais distantes dos grandes centros" 2 (p. 13).

A despeito dessas análises, que tendiam a não racializar a epidemia, simultaneamente ao lançamento do Boletim foi divulgado o Programa Integrado de Ações Afirmativas para Negros Brasil AfroAtitude ${ }^{3}$, uma parceria entre o PNDST/AIDS, a Secretaria Especial de Direitos Humanos (SEDH) da Presidência da República, a Secretaria Especial de Políticas de Promoção da Igualdade Racial (SEPPIR) e a Secretaria de Ensino Superior do Ministério da Educação. O programa previa bolsas de estudo voltadas para 
"contribuir para a formação de estudantes negros como promotores de saúde e de qualidade de vida, e para a produção de conhecimentos no campo da prevenção, aconselhamento e assistência às DST/ AIDS" 3 (p. 8).

Em 2005, também no Dia Mundial de Luta Contra a AIDS, a imprensa novamente chamou a atenção para um aumento do número de casos de AIDS entre "negros". Dessa vez, além do programa AfroAtitude, o governo elegeu a "população negra" para a sua campanha, intitulada AIDS $e$ Racismo - O Brasil tem que Viver sem Preconceito. No cartaz da campanha (Figura 1) aparece uma sorridente mulher negra, com o cabelo trançado, segurando nas duas mãos, como uma oferenda, uma camisinha. Estampadas sobre o seu peito as palavras: "Você tem direito à informação, à prevenção e ao tratamento da AIDS. Não importa a sua cor". O então Ministro da Saúde, Saraiva Felipe, declarou por ocasião do lançamento da campanha: "Resolvemos ter um olhar especial para os brasileiros afro-descendentes porque verificamos um aumento do número de casos de AIDS entre essa população. Decidimos junto com ONGs, com a Secretaria Especial de Promoção de Políticas de Igualdade Racial e com celebridades negras dar um enfoque, chamando atenção para a vinculação entre racismo, pobreza e aumento dos casos de AIDS nesse segmento da população brasileira. São pessoas que, por estarem no estrato mais pobre da sociedade, têm menos acesso às informações e aos serviços de saúde, dentro do contexto de pobre-za e discriminação racial no país" (http:// portal.saude.gov.br/portal/aplicacoes/noticias noticiasdetalhe.cfm? coseq_noticia $=22549$, acessado em 09/Mar/2006).

A partir do exposto acima, particularmente no caso das autoridades governamentais, observa-se uma mudança no discurso, com o destaque do racismo como um aspecto-chave no entendimento de uma maior vulnerabilidade da "população negra" à AIDS.

Nosso objetivo no presente trabalho é examinar o contexto sócio-político envolvido na definição de políticas públicas de recorte racial no campo da saúde no Brasil, tomando a AIDS como um estudo de caso. Argumentamos que questões ligadas à qualidade dos dados, à estruturação do sistema de informação e ao uso e interpretação das informações são elementos essenciais na compreensão do processo em curso. Especificamente, procuramos evidenciar que uma análise mais pormenorizada indica que os dados epidemiológicos disponíveis não são suficientes para sustentar a interpretação de que existe uma associação específica entre "população negra" e AIDS no país. Salientamos que a ênfase nessa associação faz parte de uma dinâmica relacionada
Figura 1

Cartaz da campanha "AIDS e Racismo - O Brasil Tem que Viver Sem Preconceito".

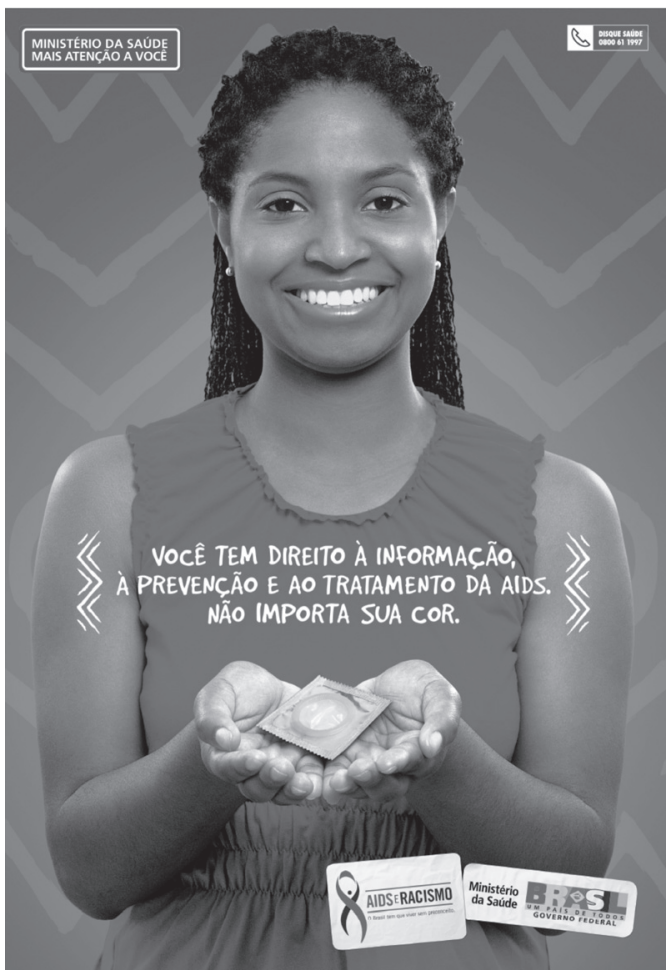

à construção do campo da "saúde da população negra” em anos recentes, que se vincula a processos mais amplos de inter-relação entre ativismo político e relação com o Estado, que transcendem a área da saúde.

\section{Cor/raça, AIDS e os sistemas de infor- mação no Brasil}

Ao contrário dos bancos de dados de outros países (como os Estados Unidos), que têm na raça uma variável fundamental, a inclusão desta variável nos grandes bancos de dados nacionais no Brasil não é só recente como ainda bastante deficiente, seja da perspectiva da utilização de classificações contrastantes nos diferentes sistemas de informação, seja da perspectiva do nãopreenchimento da variável 4,5.

No Brasil, a introdução da variável "raça" nos bancos de dados nacionais que sistematizam, direta ou indiretamente, informações referentes ao HIV/AIDS, como no Sistema de Informações de Mortalidade (SIM), em 1996, ou no Sistema de Informação de Agravos de Notificação (SINAN), 
em 2001, é tão recente que impossibilita a análise de tendências a longo prazo. São análises dessa natureza que, de fato, importam em uma perspectiva epidemiológica.

O caso da variável "cor/raça" (terminologia utilizada pelo Instituto Brasileiro de Geografia e Estatística, IBGE, e pelo PN-DST/AIDS) na base de dados sobre AIDS é exemplar das dificuldades indicadas acima. Ao se analisar o banco de dados do PN-DST/AIDS (Tabela 1), observa-se que, em 2000 , somente $3,9 \%$ dos registros apresentavam a classificação racial dos casos. Uma proporção mais expressiva de preenchimento somente aconteceu a partir de 2003, quando mais de dois terços dos casos passaram a contar com esta informação. Com proporções de respostas válidas tão baixas, qualquer interpretação se mostra uma empreitada temerosa.

Uma outra complicação no caso das análises epidemiológicas utilizando a variável "cor/raça" relaciona-se aos diferentes sistemas de classificação utilizados - auto-referida (autoclassificação) ou por terceiros (hetero-classificação) 6,7,8. Assim, enquanto "cor/raça" no banco de dados do PN-DST/AIDS deriva de classificação feita por profissionais de saúde (via de regra, médicos), na construção das taxas de prevalência de AIDS segundo "cor/raça" são utilizados nos denominadores os dados do IBGE, que derivam de autoclassificação.

Uma questão adicional da utilização da variável "cor/raça" relaciona-se à geração de estatísticas a partir da junção de certas categorias (como pardos e pretos, resultando em "negros") e seus impactos na interpretação de dados epidemiológicos. As implicações dessa junção, tomando a AIDS como referência, serão examinadas adiante.

Em suma, ainda que nos últimos anos venha se intensificando o interesse pelas análises acer- ca de "cor/raça" como fatores determinantes de desigualdades em saúde no Brasil 9,10, persistem dificuldades importantes em função da cobertura e qualidade das bases de dados, além da complexidade sócio-antropológica relativa aos sistemas de classificação 11,12,13,14.

\section{As transformações da epidemia}

Ao longo das décadas de 1980 e 1990, a epidemia de AIDS no Brasil experimentou modificações substanciais na sua dinâmica. No seu início, excepcionalmente, a AIDS afetou de forma marcante estratos médios e superiores da sociedade, a partir da introdução do vírus no país decorrente da interação de brasileiros com nacionais de países desenvolvidos (norte-americanos e europeus), afetados, à ocasião, por uma epidemia nascente.

Não por acaso, a epidemia brasileira se inicia com a disseminação de subtipos virais que, à época, predominavam na epidemia norte-americana e européia 15 , e não por intermédio de subtipos virais então predominantes no continente africano (amplamente majoritários na epidemia no nível mundial). Configurou-se, portanto, uma situação de concentração da epidemia em contingentes populacionais delimitados, particularmente das camadas média e alta. Após uma fase inicial, a epidemia brasileira só poderia trilhar dois caminhos: permanecer como uma epidemia "prevenida", como, por exemplo, na Austrália, que atingiu basicamente homossexuais masculinos numa etapa inicial, para depois declinar nesta população, sem atingir de forma relevante quaisquer outros segmentos populacionais 16; ou, como veio a ocorrer, disseminar-se para estratos mais amplos, majoritariamente desfavorecidos,

Distribuição dos casos de AIDS notificados ao SINAN-AIDS, por cor/raça. Brasil, 2000-2005.

\begin{tabular}{|c|c|c|c|c|c|c|c|c|c|c|c|c|c|c|}
\hline \multirow[t]{2}{*}{ Cor/Raça } & \multicolumn{2}{|c|}{2000} & \multicolumn{2}{|c|}{2001} & \multicolumn{2}{|c|}{2002} & \multicolumn{2}{|c|}{2003} & \multicolumn{2}{|c|}{2004} & \multicolumn{2}{|c|}{2005} & \multicolumn{2}{|c|}{ Total } \\
\hline & $n$ & $\%$ & $\mathrm{n}$ & $\%$ & $n$ & $\%$ & $n$ & $\%$ & $n$ & $\%$ & $n$ & $\%$ & $n$ & $\%$ \\
\hline Ignorado & 16.300 & 96,1 & 19.754 & 87,9 & 14.966 & 59,4 & 6.097 & 23,1 & 3.759 & 15,7 & 1.622 & 15,1 & 62.498 & 49,7 \\
\hline Branca & 416 & 2,5 & 1.743 & 7,8 & 6.702 & 26,6 & 12.397 & 46,9 & 11.467 & 47,8 & 5.033 & 46,8 & 37.758 & 30,0 \\
\hline Preta & 90 & 0,5 & 338 & 1,5 & 1.180 & 4,7 & 2.462 & 9,3 & 2.565 & 10,7 & 1.096 & 10,2 & 7.731 & 6,1 \\
\hline Amarela & 4 & 0,0 & 22 & 0,1 & 75 & 0,3 & 130 & 0,5 & 160 & 0,7 & 69 & 0,6 & 460 & 0,4 \\
\hline Parda & 150 & 0,9 & 608 & 2,7 & 2.256 & 9,0 & 5.322 & 20,1 & 5.989 & 25,0 & 2.906 & 27,0 & 17.231 & 13,7 \\
\hline Indígena & 0 & 0,0 & 6 & 0,0 & 19 & 0,1 & 37 & 0,1 & 39 & 0,2 & 19 & 0,2 & 120 & 0,1 \\
\hline Total & 16.960 & 100,0 & 22.471 & 100,0 & 25.198 & 100,0 & 26.445 & 100,0 & 23.979 & 100,0 & 10.745 & 100,0 & 125.798 & 100,0 \\
\hline
\end{tabular}

Fonte: Banco de dados do PN-DST/AIDS (http://www.aids.gov.br/cgi/deftohtm.exe?tabnet/aids.def, acessado em 27/Dez/2005). 
da população: o que a literatura descreve como "pauperização".

Encontra-se bem documentado que, no Brasil, a epidemia se disseminou entre os moradores de comunidades mais pobres, especialmente entre as mulheres 17,18, entre os de menor educação formal 19,20 e entre aqueles inseridos em ocupações mal-remuneradas ou excluídos do mercado de trabalho formal 21. Ou seja, progressivamente, a epidemia, seguindo uma tendência de outros agravos, como outras doenças infecciosas, as mortes violentas, as doenças cardíacas, as neoplasias, entre outras, passou a refletir os padrões de desigualdade predominantes no país.

Como veremos a seguir, os dados do PNDST/AIDS apontam para tendências diferentes daquelas indicadas acima.

\section{Os argumentos e os números}

O boletim epidemiológico do PN-DST/AIDS referente aos meses de janeiro a junho de 2005 começa com uma introdução, de cunho basicamente metodológico. No que diz respeito ao perfil sócio-econômico dos pacientes de AIDS, é comentado: " a epidemia de AIDS continua seu processo de crescimento entre as populações mais vulneráveis socioeconomicamente, expresso pelo aumento persistente da proporção de casos com raça/cor 'parda' e redução da 'branca', em ambos os sexos" 22 (p. 5).

O ponto central dessa afirmação é que o aumento entre os "vulneráveis socioeconomicamente" seria expresso pelo aumento dos casos entre os pardos, como se a "cor/raça”, por si só, fosse um indicador de classe. É verdade que entre os pobres, no Brasil, as proporções de pretos e pardos são elevadas, mas a afirmação de que a epidemia vem crescendo de forma particularmente acelerada entre os pobres teria de se apoiar em dados sócio-econômicos ou outros indicadores de classe, como escolaridade do pai e da mãe, por exemplo.

A única variável do banco de dados do $\mathrm{PN}$ DST/AIDS que permite informar sobre a condição sócio-econômica é a escolaridade. A Tabela 2 sumariza os dados sobre a escolaridade dos casos de AIDS entre 2000 e 2005. Dos 125.798 registros, para 22,4\% deles a variável não se encontra preenchida, considerando somente aqueles com escolaridade conhecida (Tabela 3). Pode-se perceber que a proporção de pacientes sem escolaridade formal permaneceu mais ou menos estável ao longo do período, correspondendo a $5 \%$ (Tabela 3). As porcentagens de casos de indivíduos com educação fundamental (que corresponde da 1a a 8a série do Ensino Fundamental), se reduziu pela metade no período (de $39,4 \%$ para $18,6 \%$ ). Finalmente, ocorreu substancial aumento de casos em pessoas com escolaridade média ou superior (do 1o ano do Ensino Médio ao final do Ensino Superior), ou seja, de 54,8\% para $76,4 \%$. Portanto, os dados do PN-DST/AIDS indicam que, ao contrário da afirmação sobre a pauperização que consta do boletim epidemiológico, a epidemia não estaria aumentando num ritmo proporcionalmente maior entre as populações mais vulneráveis socioeconomicamente.

Quanto ao suposto aumento de casos de AIDS entre os "afrodescendentes"/ "negros" (considerando os "negros" como a junção de pretos e pardos), os dados do PN-DST/AIDS são também reveladores. Parece haver um aumento (não linear) de casos entre "negros" no período 2000-2005 (Tabela 4; Figura 2). Em 2000 os "negros" corres-

Tabela 2

Distribuição dos casos de AIDS notificados ao SINAN-AIDS, por escolaridade. Brasil, 2000-2005.

\begin{tabular}{|c|c|c|c|c|c|c|c|c|c|c|c|c|c|c|}
\hline \multirow[t]{2}{*}{ Escolaridade } & \multicolumn{2}{|c|}{2000} & \multicolumn{2}{|c|}{2001} & \multicolumn{2}{|c|}{2002} & \multicolumn{2}{|c|}{2003} & \multicolumn{2}{|c|}{2004} & \multicolumn{2}{|c|}{2005} & \multicolumn{2}{|c|}{ Total } \\
\hline & $\mathrm{n}$ & $\%$ & $n$ & $\%$ & $n$ & $\%$ & $n$ & $\%$ & $n$ & $\%$ & $n$ & $\%$ & $n$ & $\%$ \\
\hline Ignorado * & 2.644 & 15,6 & 3.805 & 16,9 & 5.007 & 19,9 & 5.587 & 21,1 & 4.628 & 19,3 & 2.151 & 20,0 & 23.822 & 18,9 \\
\hline Ignorado ** & 269 & 1,6 & 580 & 2,6 & 1.098 & 4,4 & 1.064 & 4,0 & 963 & 4,0 & 365 & 3,4 & 4.339 & 3,4 \\
\hline Nenhuma & 827 & 4,9 & 994 & 4,4 & 1.063 & 4,2 & 1.046 & 4,0 & 893 & 3,7 & 413 & 3,8 & 5.236 & 4,2 \\
\hline Fundamental & 5.528 & 32,6 & 6.478 & 28,8 & 4.543 & 18,0 & 3.756 & 14,2 & 3.458 & 14,4 & 1.527 & 14,2 & 25.290 & 20,1 \\
\hline Médio/Superior & 7.692 & 45,4 & 10.614 & 47,2 & 13.487 & 53,5 & 14.992 & 56,7 & 14.037 & 58,5 & 6.289 & 58,5 & 67.111 & 53,3 \\
\hline Total & 16.960 & 100,0 & 22.471 & 100,0 & 25.198 & 100,0 & 26.445 & 100,0 & 23.979 & 100,0 & 10.745 & 100,0 & 125.798 & 100,0 \\
\hline
\end{tabular}

Fonte: Banco de dados do PN-DST/AIDS (http://www.aids.gov.br/cgi/deftohtm.exe?tabnet/aids.def, acessado em 27/Dez/2005).

* Informações ignoradas referentes ao SINAN-AIDS;

** Informações ignoradas referentes ao SISCEL. 
Tabela 3

Distribuição percentual dos casos de AIDS notificados ao SINAN-AIDS, por escolaridade (conhecida *). Brasil, 2000-2005.

\begin{tabular}{lcccccc}
\hline Escolaridade & $\mathbf{2 0 0 0}$ & $\mathbf{2 0 0 1}$ & $\mathbf{2 0 0 2}$ & $\mathbf{2 0 0 3}$ & $\mathbf{2 0 0 4}$ & $\mathbf{2 0 0 5}$ \\
\hline Nenhuma & 5,9 & 5,5 & 5,6 & 5,3 & 4,9 & 5,0 \\
Fundamental & 39,4 & 35,8 & 23,8 & 19,0 & 18,8 & 18,6 \\
Médio/Superior & 54,8 & 58,7 & 70,6 & 75,7 & 76,3 & 76,4 \\
\hline
\end{tabular}

Fonte: Banco de dados do PN-DST/AIDS (http://www.aids.gov.br/cgi/deftohtm.exe?tabnet/aids.def, acessado em 27/Dez/2005)

* Todos os dados faltantes foram excluídos da presente tabela.

Tabela 4

Casos de AIDS que declararam a sua cor/raça e ano de diagnóstico, por ano de notificação. Brasil, 2000-2005

\begin{tabular}{|c|c|c|c|c|c|c|c|c|c|c|c|c|c|c|}
\hline \multirow[t]{2}{*}{ Cor/Raça } & \multicolumn{2}{|c|}{2000} & \multicolumn{2}{|c|}{2001} & \multicolumn{2}{|c|}{2002} & \multicolumn{2}{|c|}{2003} & \multicolumn{2}{|c|}{2004} & \multicolumn{2}{|c|}{2005} & \multicolumn{2}{|c|}{ Total } \\
\hline & $n$ & $\%$ & $\mathrm{n}$ & $\%$ & $\mathrm{n}$ & $\%$ & $\mathrm{n}$ & $\%$ & $n$ & $\%$ & $\mathrm{n}$ & $\%$ & $n$ & $\%$ \\
\hline Branca & 416 & 63,0 & 1.743 & 64,2 & 6.702 & 65,5 & 12.397 & 60,9 & 11.467 & 56,7 & 5.033 & 55,2 & 37.758 & 59,6 \\
\hline Preta & 90 & 13,6 & 338 & 12,4 & 1.180 & 11,5 & 2.462 & 12,1 & 2.565 & 12,7 & 1.096 & 12,0 & 7.731 & 12,2 \\
\hline Amarela & 4 & 0,6 & 22 & 0,8 & 75 & 0,7 & 130 & 0,6 & 160 & 0,8 & 69 & 0,8 & 460 & 0,7 \\
\hline Parda & 150 & 22,7 & 608 & 22,4 & 2.256 & 22,0 & 5.322 & 26,2 & 5.989 & 29,6 & 2.906 & 31,9 & 17.231 & 27,2 \\
\hline Indígena & 0 & 0,0 & 6 & 0,2 & 19 & 0,2 & 37 & 0,2 & 39 & 0,2 & 19 & 0,2 & 120 & 0,2 \\
\hline Total & 660 & 100,0 & 2.717 & 100,0 & 10.232 & 100,0 & 20.348 & 100,0 & 20.220 & 100,0 & 9.123 & 100,0 & 63.300 & 100,0 \\
\hline
\end{tabular}

Fonte: Banco de dados do PN-DST/AIDS (http://www.aids.gov.br/cgi/deftohtm.exe?tabnet/aids.def, acessado em 27/Dez/2005).

Figura 2

Porcentagem de casos de AIDS em pretos, pardos e "negros" (pretos e pardos), por ano de notificação, $2000-2005$.

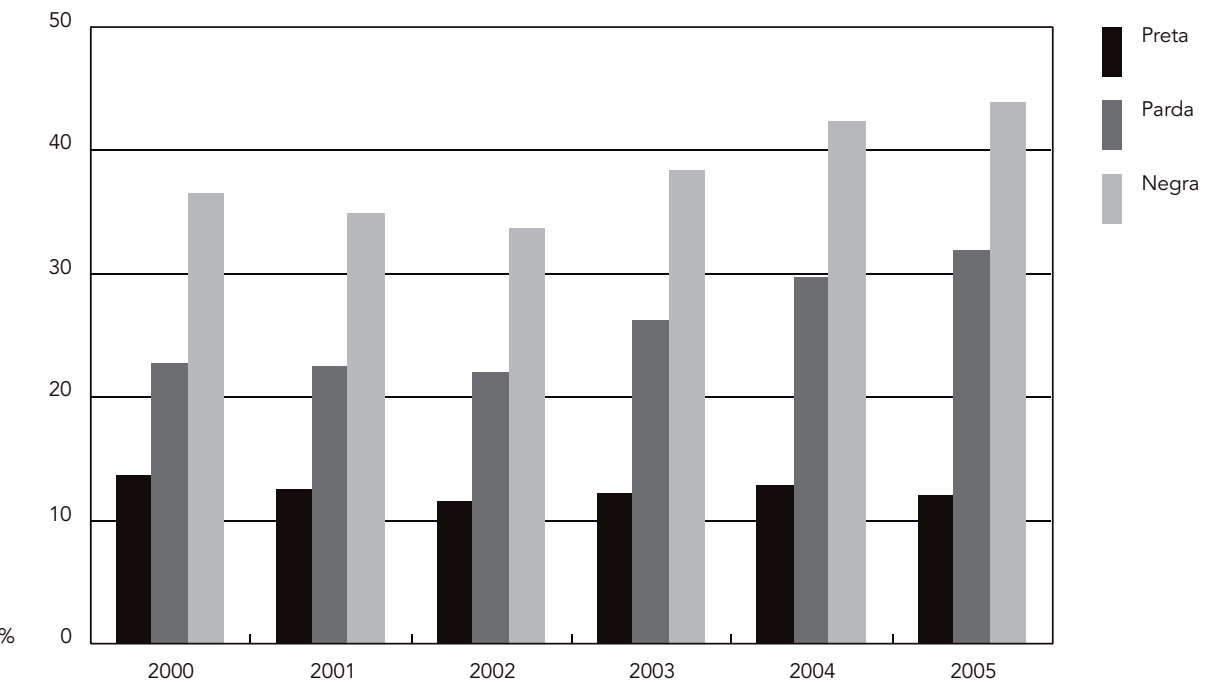


pondiam a $36,4 \%$ dos casos ( 240 em 660 ), a $34,8 \%$ em 2001 (946 em 2.717), a 33,6\% em 2002 (3.436 em 10.232), a 38,3\% em 2003 (7.784 em 20.348), a $42,3 \%$ em 2004 (8.554 em 20.220 ) e a $43,9 \%$ em 2005 (4.002 em 9.123).

Não obstante, ao se analisar as porcentagens segundo categorias de "cor/raça" específicas, identifica-se uma diferença significativa entre os casos definidos como pardos e pretos. Se, por um lado, nota-se um aumento proporcional do número de casos de AIDS entre os pardos (de 22,7\% a $31,9 \%)$, constata-se uma estabilidade entre os pretos (da ordem de 11-13\%) (Tabela 4; Figura 2). No caso dos brancos, verifica-se uma diminuição proporcional na porcentagem dos casos (de 63\% para 55,2\%) (Tabela 4 ).

Portanto, uma análise descritiva da base de dados do PN-DST/AIDS, surpreendentemente, sugere ter havido uma reversão de tendência de pauperização da epidemia de HIV/AIDS e que o suposto aumento na "população negra" resulta de um aumento somente entre os pardos, mas não entre os pretos. Esse último achado é de particular relevância, considerando que as campanhas anti-AIDS disseminadas pelo governo brasileiro estão particularmente voltadas para as pessoas de fenótipo mais escuro (Figura 1).

Frente a essas constatações resta indagar por que o governo brasileiro resolveu priorizar a "população negra" nas suas recentes campanhas anti-AIDS. Para responder a essa pergunta é necessário recuar no tempo, buscando-se compreender os contextos sócio-políticos que têm moldado as políticas públicas de recorte racial no Brasil.

\section{Recuando na história}

Em 2000, a campanha anti-AIDS para o carnaval adotou o slogan AIDS, Prevenir é Tão Fácil Quanto Pegar (Figura 3). A campanha mostrava uma mulher negra jovem, supostamente buscando o parceiro eventual do carnaval passado. Ela dizia que estava infectada com o HIV e pedia que ele fizesse um teste. "Pois não sei se peguei ou se passei o vírus para você" 23.

Lançada a campanha, não tardou para que uma organização denominada Associação Brasileira de Negros Progressistas (ABNP) a criticasse. De acordo com a matéria da Folha de S. Paulo: "A ABNP [...] pediu ao STF (Supremo Tribunal Federal) que processe o ministro José Serra [...] por crime de racismo por causa da apresentação de uma mulher negra na nova publicidade oficial sobre prevenção da AIDS. A entidade sustenta que a imagem da mulher negra está sendo ofendida por ela estar exposta no anúncio publicitário da campanha como prostituta ou 'vulgar e irresponsável' [...]. A associação é a mesma que distribuiu panfletos em vários pontos de São Paulo acusando a Folha e o Datafolha de racismo. [...] 'O governo federal recusa-se a colocar na publicidade (oficial) cidadãos negros em campanhas dignas, mantendo-os invisíveis', sustenta a associação. [...] A ABNP moveu uma representação pedindo que o Supremo inicialmente determine a instauração de inquérito policial e que depois processe e julgue Serra pelo crime de racismo. [...] A Constituição e a legislação brasileiras estabelecem que o racismo é crime inafiançável e imprescritivel..." 24

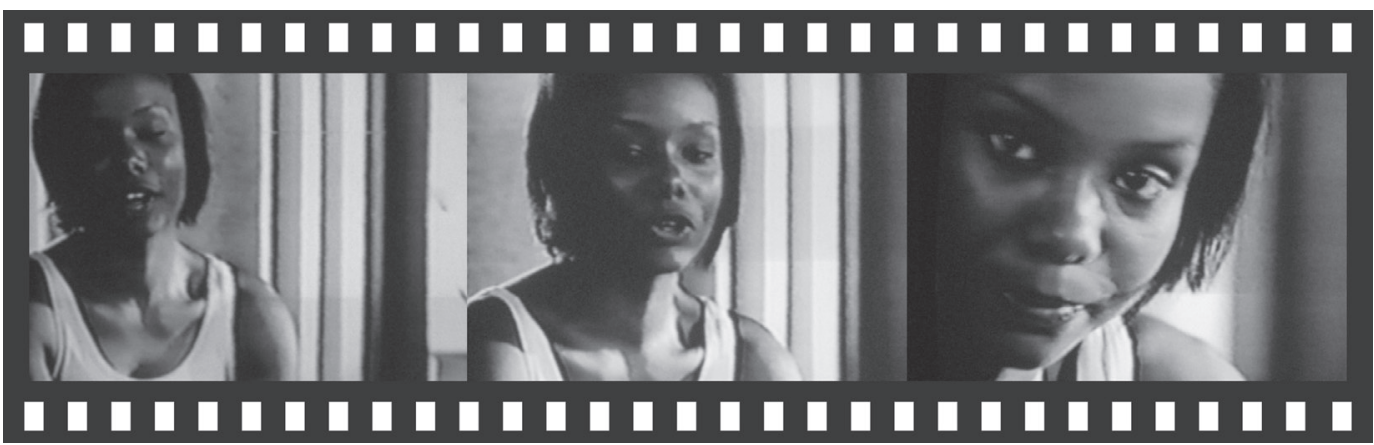

Fonte: Ministério da Saúde. 
Na ocasião, representantes governamentais se pronunciaram, defendendo a campanha: " $\mathrm{Pa}$ ra o Ministério da Saúde, a campanha não é racista. O Ministério informou ontem que a atriz foi escolhida entre 30 candidatas, grupo que incluía louras, morenas e negras, e que só teria ocorrido racismo se a melhor candidata não pudesse estrelar a campanha pelo fato de ser negra" 24.

Um mês depois, um jornalista da Folha de $S$. Paulo entrevistou três mulheres ativistas negras e a atriz da campanha 25. Elas se manifestaram de forma bastante distintas.

Nilza Iraci, da Rede Eletrônica de Comunicação de Mulheres Negras (RECON), em conformidade com a ABNP, criticou os estereótipos associados às mulheres negras: “Na década de 70, o movimento feminista denunciava a representação das mulheres, divididas entre 'santas e putas'. A negra nunca foi representada na 'santidade'; sua construção social aconteceu pelo corpo: a mais sexual, lasciva e disponivel, ou como a 'mãe preta', um grande peito que sustenta vidas alheias. Os estereótipos ainda continuam. Se queriam uma campanha eficiente deveriam ter consultado especialistas do Movimento Negro para aproveitar melhor o dinheiro público" 25 (p. 38).

Na mesma linha, Sueli Carneiro, coordenadora executiva do Instituto da Mulher Negra (Geledés), sugeriu que não era mera coincidência a escolha de uma atriz negra para o papel da mulher com AIDS, minimizando o risco associado à epidemia no contexto dos demais agravos de saúde e problemas sociais: "O que causa 'estranhamento' éo 'ineditismo' de uma campanha institucional ser conduzida por uma negra retinta, que embora bela e competente atriz, nunca foi cogitada anteriormente para ancorar campanhas pela erradicação do analfabetismo, de combate à miséria, à desnutrição, pela assistência humanizada ao parto, contra o desemprego ou a violência social, temas que são tão ou mais dizimadores do que a epidemia da AIDS” 25 (p. 38).

Já Edna Roland, presidente da Organização de Mulheres Negras (Fala Preta!), e em um momento posterior relatora da 3a Conferência Mundial das Nações Unidas de Combate ao Racismo, Discriminação Racial, Xenofobia e Intolerância Correlata que teve lugar em Durban, na África do Sul, em 2001, apoiou o conteúdo da campanha do governo. Na sua visão, "A mensagem é precisa e apresenta a negra de forma digna. E ainda chama a atenção desse setor da população que enfrenta dificuldade de acesso à saúde e educação. É lamentável a posição retrógrada da Associação dos Negros Progressistas. Para que a AIDS não seja mais um fator de extermínio do povo negro neste país, precisamos de informações e posturas livres de preconceitos" 25 (p. 38).
Finalmente, a atriz em questão, Carla Leite, insistiu que a AIDS não é um "problema racial". Para ela, tratar-se-ia de uma questão de informação: "[u] ma campanha como essa tem que ser vista pelo lado da conscientização e só. Tenho certeza que não desfavorece em nada a imagem da mulher negra. Se estou arrependida? Não, muito pelo contrário!" 25 (p. 38). Numa outra entrevista, a atriz foi mais enfática na defesa da sua posição: “Essa campanha existe há anos e só agora colocaram um rosto negro. A imagem não está vinculada à prostituição ou à promiscuidade. A AIDS não tem cor, idade ou condição social" 26 (p. 18).

Se a campanha de 2000 não priorizava a relação entre "cor/raça" e AIDS, a de 2005, como vimos no início deste trabalho, foi montada a partir de uma ênfase na especificidade da "população negra” em relação à AIDS (Figura 1). Por que em 2005 não se criticou a campanha, ainda que a mesma fosse centrada numa modelo negra? Por que ninguém alertou, tal como em 2000, para uma possível associação entre AIDS e pessoas "negras", aumentando, com isso, a possibilidade de preconceito e discriminação?

O que mudou entre 2000 e 2005 foi a pauta governamental sobre a questão racial no Brasil. Tais mudanças revelam que o Estado se tornou crescentemente permeável às demandas dos movimentos sociais, em particular o movimento negro, bem como as dinâmicas internacionais na área dos direitos humanos.

A inflexão começou no governo de Fernando Henrique Cardoso, com o reconhecimento oficial da existência do racismo no Brasil. Na sua gestão foi criado o Grupo de Trabalho Interministerial (GTI) para a Valorização da População Negra, por ocasião de uma manifestação em homenagem aos 300 anos de Zumbi dos Palmares, em novembro de 1995. Essa iniciativa veio no bojo do Programa Nacional de Direitos Humanos (PNDH) do Governo Federal e da ampliação do debate sobre a implementação de políticas de ação afirmativa no Brasil 13,27,28.

Nesse contexto, conforme revelam as análises de Fry 29,30 e Maio \& Monteiro 9, estabeleceu-se um GTI voltado particularmente para a reflexão e a definição de propostas de intervenção acerca das interfaces entre saúde e "população negra". Uma das primeiras iniciativas desse grupo foi organizar, em 1996, uma mesa-redonda sobre o tema com profissionais da saúde, acadêmicos, ativistas e técnicos do Ministério da Saúde. Nesse evento se definiu um conjunto de agravos supostamente associados à população negra, entre eles: as doenças geneticamente determinadas, com destaque para a anemia falciforme; as enfermidades advindas da combinação entre aspectos genéticos e condições sócio-econômicas adver- 
sas (ex.: hipertensão arterial, miomas) e os males provocados pelas precárias condições de vida (ex.: alcoolismo, alta mortalidade infantil, AIDS) (http://www.planalto.gov.br/pwubli_04/colecao/racial.2h.htm, acessado em 23/Jan/2004).

A 3a Conferência Mundial de Combate ao Racismo, ocorrida em Durban, em 2001, determinaria alterações importantes na construção de "políticas raciais" no campo da saúde. Diversas conferências regionais e mundiais serviram de catalisadores para a inserção da discussão sobre o racismo na pauta de questões nacionais. Esse contexto deixou mais evidente as articulações entre ONGs, agências internacionais de financiamento e aparatos estatais no âmbito da luta pelos direitos humanos. Jornalistas, economistas, setores da academia e parlamentares de variados matizes ideológicos passaram a apoiar políticas públicas racializadas como forma de se atingir justiça social, em contraposição às de perfil universalista 27

No campo da saúde pública, em dezembro de 2001, profissionais de várias organizações internacionais, do movimento negro e de instituições universitárias produziram o documento Subsídios para o Debate sobre a Política Nacional de Saúde da População Negra. Uma Questão de Eqüidade 31. Tal política é justificada a partir de diversos argumentos, tais como: de que a "discriminação racial leva a situações mais perversas de vida e de morte"; de que há desigualdades importantes entre "negros" e brancos, e de que a universalidade dos serviços, garantida pelo Sistema Único de Saúde (SUS), não seria suficiente para "assegurar a eqüidade [...] ao subestimar as necessidades de grupos populacionais específicos", colaborando para o agravo "das condições sanitárias de afro-brasileiros” 31 (p. 6-7). O documento reitera os principais pontos da MesaRedonda sobre a Saúde da População Negra, de 1996.

Com a eleição de Luis Inácio Lula da Silva houve a ampliação das políticas destinadas à "população negra", sobretudo com a criação da Secretaria Especial de Promoção de Políticas para Igualdade Racial (SEPPIR), em 2003. Em agosto de 2004, foi constituído o Comitê Técnico de Saúde da População Negra. Nessa ocasião ocorreu o 1o Seminário Nacional da Saúde da População Negra em Brasília, patrocinado pelo Ministério da Saúde e pela SEPPIR. Um dos desdobramentos desse evento foi o lançamento do Programa Brasil AfroAtitude que, como vimos, visa a estimular projetos e estudos sobre a vulnerabilidade ao HIV/AIDS da "população negra" por meio da concessão de bolsas a alunos cotistas de cursos de graduação de universidades públicas 9,30.
Novas iniciativas nessa direção estão descritas no Programa Estratégico de Ações Afirmativas: População Negra e AIDS, desenvolvido pelo PNDST/AIDS, SEPPIR, SEDH, MEC e Ministério da Saúde, e lançado em agosto de 2005. Apoiado no pressuposto de que o racismo, o sexismo e a homofobia "são fatores de produção de vulnerabilidade ao HIVIAIDS para pessoas e comunidades da população negra" 32 (p. 10), o programa, composto por 31 metas, tem como principal objetivo a promoção da eqüidade e dos direitos humanos da população negra, que incluem a facilitação do acesso deste grupo ao SUS e a organização e disseminação das informações disponíveis acerca da saúde da população negra no Brasil 32 (p. 10-1). Integram as ações do programa: a inserção de jovens negros no programa Saúde e Prevenção nas Escolas; a realização de campanhas específicas sobre AIDS, racismo, outras DST e a "população negra”; a participação de $50 \%$ de pessoas negras nas campanhas do PN-DST/AIDS; a implementação de projetos para a população quilombola. Vale destacar que parte significativa do programa é composta por verbetes e textos sobre ação afirmativa, apartheid, cotas, eqüidade, diversidade, discriminação (racial), direitos humanos, mestiçagem, meritocracia, racismo, política universalista, desigualdades entre brancos e negros e AIDS, quesito cor no sistema de informação em saúde, entre outros. Esses conceitos e abordagens revelam o caráter pedagógico do programa, voltado fundamentalmente para o processo de conscientização racial.

Portanto, vê-se que a lógica dos programas voltados para a "saúde da população negra" tem o pressuposto de que os brasileiros podem e devem ser classificados em duas categorias estanques ("brancos" e "negros"), cada qual com suas especificidades no campo da saúde. A ênfase na associação entre "raça" e saúde pode ter como conseqüência a "naturalização" e o fortalecimento de supostas diferenças raciais entre os grupos populacionais, minimizando a gênese social e histórica das mesmas. Em outras palavras, nossa interpretação é de que o Programa Estratégico de Ações Afirmativas: População Negra e AIDS faz parte de uma estratégia política de racializar um agravo à saúde (e, no caso específico, a dinâmica de uma epidemia), com vistas a fortalecer identidades com contornos raciais. Conforme tem sido enfatizado na literatura antropológica e sociológica, um dos desafios do movimento negro no Brasil é produzir na população uma "consciência negra", buscando romper com o paradigma da mestiçagem e acumular forças com vistas a influenciar os rumos das políticas públicas 28,29 


\section{Considerações finais}

Há várias ironias e paradoxos no processo aqui descrito. Nos primórdios da epidemia de AIDS, no Brasil e em outras partes do mundo, empregava-se o conceito de "grupo de risco" para focalizar determinadas categorias e grupos sociais, como os homossexuais, os africanos, os haitianos, os profissionais do sexo 33,34. Posteriormente, foi empreendido um enorme esforço, intelectual e político, para dissociar a nascente epidemia de identidades sociais/raciais/sexuais específicas. Esse esforço se justificava por duas razões, de ordem prática e moral. Arazão prática se baseava no conhecimento sobre o processo de disseminação do HIV através do contato com o sangue, sêmen e secreção vaginal contaminados, independentemente do status dos indivíduos. A segunda razão, de ordem moral, era evitar a estigmatização de determinadas categorias, associando-as à epidemia e à síndrome clínica por meio do conceito de "grupo de risco".

O PN-DST/AIDS, ao enfatizar a maior vulnerabilidade dos "negros" ao HIV/AIDS, parece recuar no tempo na medida em que vincula identidades raciais a "grupos de risco". Essa ironia fica ainda mais evidente quando se compara o programa de DST-AIDS brasileiro com seu congênere na África do Sul. Ao compararem a política anti-AIDS do Brasil e da África do Sul, Gauri \& Lieberman 35 atribuem o relativo sucesso do programa brasileiro, sobretudo, ao fato do governo ter encarado a epidemia de AIDS como uma ameaça nacional, implementando políticas focadas crescentemente nas práticas sexuais, antes que nas identidades em geral, e nunca, até 2004, nas identidades "raciais": "Despite racial differences in Brazil, and an increasingly open discussion about general socio-economic differences along skin color lines in recent years particularly as researchers demonstrate the association between race and economic opportunity/position in that society, strong historical legacies continue to prevent race from becoming a 'politicizable' dimension of the political conflict over policy" 35 (p. 30).

Por sua vez, na África do Sul a racialização do debate sobre AIDS era tamanha, a ponto de membros de cada grupo "racial" acusar os demais pela disseminação da epidemia, que não foi possível desenvolver uma política nacional 35 .

Não estamos sugerindo que a mais recente campanha governamental de prevenção do HIV/
AIDS, focada na temática racial, possa prejudicar o esforço nacional contra a epidemia. Esse programa, de amplo reconhecimento internacional, continua a se pautar nos princípios da universalidade, eqüidade e integralidade que norteiam as políticas públicas na área da saúde no Brasil. Não obstante, a racialização da AIDS é, por certo, um novo elemento (e, afirmaríamos, preocupante) na complexa dinâmica epidemiológica e sóciopolítica da epidemia.

A análise dos dados oficiais da AIDS disponíveis é surpreendente na medida em que contraria a tendência de crescimento de casos entre as populações mais vulneráveis socioeconomicamente, apontada em vários estudos epidemiológicos 18,19,20,21. Revela também que a junção dos casos de HIV/AIDS de pardos e pretos na categoria "negra” gera um cenário epidemiológico que não é representativo do comportamento da epidemia ao se considerar os grupos de raça/cor específicos: ou seja, homogeneíza um conjunto cujas partes são individualmente heterogêneas. Assim, ainda que se tenha enfatizado o crescimento da AIDS entre os "negros", no que se refere aos pretos observou-se uma estabilidade no número de casos ao longo dos anos. Frisamos neste trabalho que esse fato tem implicações quando se considera a definição dos conteúdos e das estéticas adotados nas campanhas de prevenção.

Para além de sugerir um problema no sistema de informação, procuramos demonstrar também que as contradições entre os números e os argumentos que apóiam a definição do programa em foco, pode ser melhor compreendida por meio da contextualização sócio-política das mudanças recentes nos discursos e na produção de dados sobre as alegadas especificidades da "saúde da população negra”.

Em seu clássico livro A Falsa Medida do Homem 36 , que é uma crítica contundente ao determinismo biológico e racial na história da ciência, Stephen Jay Gould nos adverte, a um só tempo, sobre a posição privilegiada adquirida pelos dados quantitativos no mundo moderno e a potencial falácia dos números quando não se atenta para as influências sócio-políticas dos contextos nos quais são produzidos e interpretados. Em nossa opinião, os dados disponíveis do PN-DST/ AIDS não embasam, de forma consistente, o desenvolvimento de políticas públicas de recorte racial voltadas para o controle da epidemia, conforme tem sido proposto no Brasil. 


\section{Resumo}

Nos últimos anos observa-se uma ênfase numa suposta associação entre a epidemia de AIDS e a "população negra" no Brasil. Após proceder uma análise do banco de dados sobre a ocorrência de HIVIAIDS no Brasil, o presente estudo examina o contexto sóciopolítico envolvido na definição de políticas públicas de recorte racial no campo da saúde. Argumentamos que questões ligadas à qualidade dos dados, à estruturação do sistema de informação e ao uso e interpretação das informações são elementos essenciais na compreensão do processo em curso. Especificamente, procuramos mostrar que os dados epidemiológicos disponíveis não são suficientes para sustentar a interpretação de que existe uma associação específica entre "população negra" $e$ AIDS no país. Salientamos que a ênfase nessa suposta associação faz parte de uma dinâmica relacionada à construção do campo da "saúde da população negra" em anos recentes, que se vincula a processos mais amplos de inter-relação entre ativismo político e relação com o Estado, que transcendem a área da saúde.

Síndrome de Imunodeficiência Adquirida; Políticas Públicas; Racismo

\section{Referências}

1. Weber D. Aids cresce entre negros e pardos mais pobres. O Globo 2004; 1 dez.

2. Campbell U. A nova cara da AIDS. Correio Braziliense 2004; $1 \mathrm{dez}$.

3. Presidência da República/Programa Nacional de DST/AIDS, Secretaria de Vigilância em Saúde, Ministério da Saúde/Secretaria Especial dos Direitos Humanos/Conselho Nacional de Combate à Discriminação. AfroAtitude: programa integrado de ações afirmativas para negros. Brasília: Presidência da República/Ministério da Saúde; 2004.

4. Cardoso AM, Santos RV, Coimbra Jr. CEA. Mortalidade infantil segundo raça/cor no Brasil: o que dizem os sistemas nacionais de informação? Cad Saúde Pública 2005; 21:1602-8.

5. Travassos C, Williams DR. The concept and measurement of race and their relationship to public health: a review focused on Brazil and the United States. Cad Saúde Pública 2004; 20:660-78.

6. Caetano AJ. A relação entre cor da pele/raça e esterilização no Brasil: análise dos dados da pesquisa nacional sobre demografia e saúde-1996. In: Monteiro S, Sansone L, organizadores. Etnicidade na América Latina: um debate sobre saúde, raça e direitos reprodutivos. Rio de Janeiro: Editora Fiocruz; 2004; p. 229-47.

7. Carvalho JMD. Genocídio racial estatístico. O Globo 2004; $27 \mathrm{dez}$.

\section{Colaboradores}

Todos os autores participaram das etapas de concepção, análise, redação e revisão final do manuscrito.

\section{Agradecimentos}

Agradecemos a Eduardo Pina, da equipe editorial de Cadernos de Saúde Pública, pela ajuda na preparação das imagens das campanhas.
8. Romero DE, Cunha CB. Avaliação da qualidade das variáveis sócio-econômicas e demográficas dos óbitos de crianças menores de um ano registrados no Sistema de Informações sobre Mortalidade do Brasil (1996/2001). Cad Saúde Pública 2006; 22:673-81

9. Maio MC, Monteiro S. Tempos de racialização: o caso da "saúde da população negra" no Brasil. Hist Ciênc Saúde-Manguinhos 2005; 12:419-46.

10. Monteiro S. Desigualdades em saúde, raça e etnicidade: questões e desafios. In: Monteiro S, Sansone L, organizadores. Etnicidade na América Latina: um debate sobre saúde, raça e direitos reprodutivos. Rio de Janeiro: Editora Fiocruz; 2004. p. 45-56.

11. Harris M, Consorte JG, Lang J, Byrne B. Who are the whites? Imposed census categories and the racial demography of Brazil. Soc Forces 1993; 72:451-62.

12. Nogueira O. Preconceito racial de marca e preconceito racial de origem (sugestão de um quadro de referência para a interpretação do material sobre relações raciais no Brasil). In: Anais do XXXI Congresso Internacional dos Americanistas. São Paulo: Editora Anhembi; 1955. p. 409-34.

13. Fry P. Politics, nationality, and the meanings of "race"' in Brazil. Daedalus 2000; 129:83-118.

14. Maggie Y. "Aqueles a quem foi negada a cor do dia": as categorias cor e raça na cultura brasileira. In: Maio MC, Santos RV, organizadores. Raça, ciência e sociedade. Rio de Janeiro: Editora Fiocruz; 1996. p. 208-18. 
15. Bello G, Guimarães ML, Morgado MG. Evolutionary history of HIV-1 subtype B and F infections in Brazil. AIDS 2006; 20:763-8.

16. Des Jarlais DC, Hagan H, Friedman SR, Friedmann P, Goldberg D, Frischer M, et al. Maintaining low HIV seroprevalence in populations of injecting drug users. JAMA 1995; 274:1226-31.

17. Guimarães CD. Aids no feminino: por que a cada dia mais mulheres contraem Aids no Brasil? Rio de Janeiro: Editora UFRJ; 2001.

18. Szwarcwald CL, Bastos FI, Esteves MAP, Andrade CLT. A disseminação da epidemia da AIDS no Brasil, no período de 1987-1996: uma análise espacial. Cad Saúde Pública 2000; 16 Suppl 1:7-19.

19. Fonseca MG, Bastos FI, Derrico M, Andrade CLT Travassos C, Szwarcwald CL. AIDS e grau de escolaridade no Brasil: evolução temporal de 1986 a 1996. Cad Saúde Pública 2000; 16 Suppl 1:77-87.

20. Fonseca MGP, Szwarcwald CL, Bastos FI. Análise sociodemográfica da epidemia de Aids no Brasil, 1989-1997. Rev Saúde Pública 2002; 36:678-85.

21. Fonseca MGP, Travassos C, Bastos FI, Silva NV, Szwarcwald CL. Distribuição social da AIDS no Brasil, segundo participação no mercado de trabalho, ocupação e status sócio-econômico dos casos de 1987 a 1998. Cad Saúde Pública 2003; 19:1351-63.

22. Chequer P. Apresentação. Boletim Epidemiológico - AIDS 2005; Ano II, n.1.

23. Anônimo. Programa de AIDS amplia cobertura. Folha de S. Paulo 2000; 6 dez.

24. Anônimo. Campanha é acusada de ser preconceituosa. Folha de S. Paulo 2000; 2 mar.

25. Anderson J. Mídia, AIDS e preconceitos. Folha de S. Paulo 2000; 2 abr.

26. Castelo-Branco A. AIDS não tem cor, idade ou condição social. O Dia 2000; 2 mar.

27. Htun M. From racial democracy to affirmative action: changing state policy on race in Brazil. Lat Am Res Rev 2004; 39:60-89.
28. Maio MC, Santos RV. Políticas de cotas raciais, os "olhos da sociedade" e os usos da antropologia: o caso do vestibular da Universidade de Brasília (UnB). Horizontes Antropológicos 2005; 23:181214.

29. Fry P. A persistência da raça. São Paulo: Civilização Brasileira; 2005.

30. Fry P. O significado da anemia falciforme no contexto da "política racial" do governo brasileiro 1995-2004. Hist Ciênc Saúde-Manguinhos 2005; 12:347-70.

31. Programa das Nações Unidas para o Desenvolvimento. Subsídios para o debate sobre a "Política Nacional de Saúde da População Negra. Uma questão de eqüidade". Brasília: Programa das Nações Unidas para o Desenvolvimento; 2001.

32. Programa Nacional de DST-AIDS, Ministério da Saúde/Secretaria Especial de Direitos Humanos/ Secretaria Especial de Políticas de Promoção da Igualdade Racial/Secretaria de Ensino Superior, Ministério da Educação. Programa estratégico de ações afirmativas: população negra e Aids. Brasília: Ministério da Saúde; 2005.

33. Fee E, Fox D, editors. AIDS: the making of a chronic disease. Berkeley: University of California Press; 1992.

34. Farmer P. AIDS and accusation: Haiti and the geography of blame. Berkeley: University of California Press; 1993.

35. Gauri V, Lieberman ES. AIDS and the state: the politics of government responses to the epidemic in Brazil and South America. In: Annual Meetings of the American Political Science Association. http://comparativepolitics.stanford.edu/ Papers2004-05/Liebermanv2.440dJan17.pdf (acessado em 29/Jun/2006).

36. Gould SJ. A falsa medida do homem. Rio de Janeiro: Editora Martins Fontes; 1991.

Recebido em 18/Jul/2006

Aprovado em 19/Jul/2006 